Paru dans : G. Corminboeuf, M.-J. Béguelin (sous la dir. de), Du système linguistique aux actions langagières. Mélanges en l'honneur d'Alain Berrendonner. Bruxelles : De Boeck-Duculot, 2011, 249-261.

\title{
Déjà en emploi justificatif
}

\author{
Denis Apothéloz \\ Université de Nancy 2 et laboratoire ATILF \\ Małgorzata Nowakowska \\ Université Pédagogique de Cracovie (Pologne)
}

\section{Introduction}

L'emploi de l'adverbe déjà que nous nous proposons d'examiner ici a été repéré depuis longtemps dans la littérature sur les adverbes et a reçu divers qualificatifs : "logique» (Martin 1980), «argumentatif» (Paillard, s.d.) et « connectif» (Mosegaard Hansen 2008), notamment. Cependant, il est généralement mentionné assez rapidement, relégué dans le domaine des faits oraux et n'a donné lieu, à notre connaissance, à aucune étude particulière. Par ailleurs, son identification même paraît entourée d'une certaine confusion, qui tient probablement au fait que sa position dans l'énoncé varie davantage qu'on ne l'écrit habituellement. On retrouve parfois cette même confusion dans les typologies qui ont été proposées des emplois de déjà. Quoi qu'il en soit, il nous a paru que le lien qu'entretient déjà justificatif avec la dimension argumentative du discours méritait qu'on lui consacre une petite étude. Tel est le but du présent article. suivants ${ }^{1}$ :

On entendra ici par "justificatifs" les emplois de déjà illustrés par les exemples

(1) De Pradts aurait pu ne pas te le répéter, et surtout en le déformant. Déjà je barbouille toujours un peu quand je parle; s'il faut encore que ce soit déformé quand on le répète... (Montherlant, 1951, F)

(2) J'aime pas ceux qui nagent avec des palmes à la piscine. Déjà que je n'avance pas, ils me flanquent des complexes. (Méril, 1997, F)

(3) C. Allègre ne maîtrisant déjà pas l'acronyme G.I.E.C., faut-il porter attention au reste de son ouvrage? (Titre de rubrique, journal Internet)

A titre de comparaison, on observera que dans ces formulations, l'effet sémantique auquel déjà est associé diffère très sensiblement de celui qu'on rencontre dans les emplois suivants (liste non exhaustive) $)^{2}$ :

\footnotetext{
${ }^{1}$ Les exemples notés ' $\mathrm{F}$ ' ont été trouvés grâce à la base de données Frantext, hébergée à l'ATILF.

${ }^{2}$ Pour une réflexion typologique plus approfondie, voir notamment Muller (1975), Hoepelman \& Rohrer (1980), Martin (1980), Fuchs (1988), Franckel (1989), Paillard (1992, s.d.), Nøjgaard (1992-95), Mosegaard Hansen (2002, 2008), Tahara (2006), Buchi (2007), Métrich et al. (2009 et à par.), Apothéloz \& Nowakowska (à par.). Voir aussi König (1977) et Métrich et al. (à par.) pour l'allemand schon, van der Auwera (1993) pour l'anglais already, et Nowakowska \& Apothéloz (2011) pour le polonais już.
} 
- de survenance précoce : Il est déjà midi,

- factuels ou associés à un parfait d'expérience au sens de Comrie (1976), cas dans lesquels la présence de l'adverbe ne modifie pas vraiment le sens de l'énoncé : Avezvous déjà commandé ?, J'ai déjà été en prison,

- itératifs : Il faisait déjà ce bruit hier,

- catégoriels : Un poing c'est déjà une arme,

- prospectifs : Je vais déjà lui écrire,

- mémoriels : C'est comment déjà votre nom?

- de minimisation, que König (1977) glose par «Don't worry » : Nous trouverons déjà de l'argent.

Il parait intuitivement clair que dans le contexte des exemples (1)-(3), la valeur associée à déjà diffère passablement de celle des exemples ci-dessus. En première approximation, on peut dire que dans (1)-(3), l'adverbe contribue à donner un statut discursif particulier au fait désigné par l'énoncé dans lequel il figure. Il laisse par ailleurs entendre que d'autres faits pourraient être évoqués dans le cadre de la justification de la même conclusion. A savoir : dans (1), d'autres faits que 'je barbouille toujours un peu quand je parle'; dans (2), que 'je n'avance pas quand je nage'; dans (3), que 'C. Allègre ne maîtrise pas l'acronyme G.I.E.C.'.

Le propos de cet article est donc d'étudier les propriétés discursives et argumentatives de l'adverbe déjà tel qu'il est employé dans les exemples (1)-(3). Pour ce faire, il s'agira en particulier de mettre en œuvre diverses notions issues du champ de l'argumentation.

Petite remarque avant de commencer cette étude : on aura remarqué que nous ne parlons jamais de telle ou telle "valeur» ni de tel ou tel «sens" de déjà. C'est que nous sommes convaincus, à l'instar par exemple de Franckel (1989), que le fonctionnement de cet adverbe peut s'expliquer à partir d'un unique ensemble de propriétés, et que les divers effets de sens qu'il est susceptible de produire tiennent seulement à la manière dont ces propriétés interagissent avec l'environnement sémantique et pragmatique dans lequel se trouve l'adverbe. En d'autres termes, nous pensons que les approches polysémiques de déjà s'exposent à une prolifération non contrôlée de valeurs et, surtout, passent à côté de généralisations descriptivement des plus intéressantes. Ce point est développé dans Apothéloz \& Nowakowska (à par.).

\section{Déjà justificatif : description}

Essayons de dresser un rapide bilan des principales propriétés de déjà tel qu'il est utilisé dans les exemples (1)-(3).

1. Il indique que le contenu auquel il est associé est lié à une argumentation, autrement dit à l'articulation de deux contenus propositionnels, dont l'un a le statut de conclusion et l'autre (celui auquel déjà est associé), le statut de justification donnée en faveur de cette conclusion. Plus exactement, déjà justificatif caractérise le statut de la séquence qui est sous sa portée comme ayant une fonction de justification. Comme on le verra, les contenus correspondant à la conclusion et à la justification ne sont pas toujours formulés de façon complète. La conclusion peut par exemple être totalement implicite, auquel cas l'adverbe marque seulement que le contenu auquel il est associé possède une orientation argumentative particulière ${ }^{3}$. Pour cette raison, l'interprétation d'un énoncé comportant cet emploi de déjà

\footnotetext{
${ }^{3}$ Notion bien évidemment empruntée aux travaux d'Anscombre et de Ducrot (cf. par ex. Anscombre et Ducrot 1976, Ducrot 1980).
} 
sollicite fortement les activités inférentielles et les principes pragmatiques habituellement convoqués quand il y a implicite, avec tous les effets d'ambiguïté et d'indétermination sémantique que cela peut impliquer.

Tentons d'appliquer aux exemples (1)-(3) ce qui vient d'être dit.

Dans (1), 'je barbouille toujours un peu quand je parle' se voit attribuer par déjà un statut de justification. Le contenu en direction duquel cette justification est dirigée (la conclusion) pourrait être reconstruit comme 'il ne faut pas déformer ce que je dis'.

Dans (2), déjà fait de 'je n'avance pas quand je nage' un fait à valeur justificative. La conclusion que ce fait vise à accréditer peut être formulée comme 'je n'aime pas ceux qui nagent avec des palmes à la piscine', ou 'ceux qui nagent avec des palmes à la piscine vont tellement vite qu'ils me flanquent des complexes'.

Dans (3), le fait suivant lequel 'C. Allègre ne maitrise pas l'acronyme G.I.E.C.' est une justification. La conclusion visée par celle-ci doit être cherchée dans la question qui suit, de toute évidence rhétorique, et peut être formulée ainsi : 'il ne vaut pas la peine de porter attention à l'ouvrage de C. Allègre'.

2. Vu sous l'angle des mécanismes interprétatifs qu'il déclenche, déjà justificatif invite à construire un gradient de faits justificatifs (se limitant la plupart du temps à deux faits, celui que met en évidence déjà, et un autre) : non seulement notre déjà signale que le contenu propositionnel sur lequel il porte est donné comme justification en faveur d'une certaine conclusion $^{4}$, mais il laisse également entendre que d'autres justifications pourraient être évoquées en faveur de la même conclusion - tout en indiquant que la justification à laquelle l'adverbe est associé a une certaine priorité sur les autres. Cette priorité n'est pas nécessairement, comme cela a parfois été écrit, celle de «meilleur argument», ni même d'argument décisif; bien plus souvent il s'agit seulement de marquer la justification comme la première qui vient à l'esprit, voire plus trivialement la première qui est énoncée ${ }^{5}$. Mais parfois cette priorité peut s'accompagner d'une certaine antériorité logique, causale ou temporelle. Tel est précisément le cas de (1), dont on peut maintenant compléter l'analyse ainsi (avec les nuances d'usage dans ce type de reconstruction) :

- conclusion: 'il ne faut pas déformer ce que je dis',

- première justification: 'je barbouille toujours un peu quand je parle',

- seconde justification : 'on a déformé mes propos'.

Mais, dans tous les cas, il semble bien que la justification soit présentée comme suffisante, ainsi que le notent Métrich et al. (à par.). Les autres justifications sont parfois implicites, comme dans (2)-(3), si on accepte les analyses données plus haut; mais elles peuvent aussi être expressément désignées, comme dans (1). Dans ce cas, elles sont assez souvent introduites par une expression comme alors si en plus, encore, etc., d'où des constructions corrélatives caractéristiques, du type :

\footnotetext{
${ }^{4}$ Que ce déjà caractérise comme justificative la fonction d'un certain contenu propositionnel ne signifie pas pour autant que tout déjà en tête d'un énoncé justificatif soit lui-même justificatif. Examinons l'exemple suivant :

Regarde comme je suis grande : déjà je peux lancer le javelot, et je sais aussi toucher le but avec ma fronde.

(Gracq, 1954, F)

Dans cet exemple, je peux lancer le javelot est donné comme justification en faveur de je suis grande. Pourtant déjà est ici très vraisemblablement, en dépit de sa position initiale, un adverbe purement temporel signifiant la survenance précoce (il présuppose qu'avant je ne savais pas encore lancer le javelot).

${ }^{5}$ Déjà justificatif a souvent été décrit comme non-temporel, pour l'opposer à d'autres emplois. Mais on peut se demander si la temporalité n'est pas simplement déplacée ici au niveau de l'énonciation, comme en atteste la proximité sémantique entre déjà justificatif et d'abord.
} 
- déjà $\mathrm{X}$, s'il faut encore que $\mathrm{Y}$

- déjà $\mathrm{X}$, et voilà encore que $\mathrm{Y}$

- déjà $\mathrm{X}$, alors si (en plus) $\mathrm{Y}$

- déjà $\mathrm{X}$, (et) en plus $\mathrm{Y}$

- déjà $\mathrm{X}$, et puis $\mathrm{Y}$

- déjà $\mathrm{X}$, et maintenant $\mathrm{Y}$

- etc.

Nous appellerons corrélateurs les expressions s'il faut encore que, alors si en plus, et en plus, etc., qui introduisent cette justification supplémentaire et indiquent qu'elle est orientée vers la même conclusion que la justification signalée par déjà.

3. Au plan énonciatif, déjà justificatif est externe, «méta-énonciatif». Son fonctionnement s'apparente ainsi à celui d'une conjonction ou d'un connecteur - Mosegaard Hansen (2008) parle de connective use, Fernandez (1994) y voit une particule énonciative. Ce statut particulier explique pourquoi il figure fréquemment en position initiale, comme dans (1)-(2), et qu'il peut introduire une question, comme dans (4) :

(4) [Réponse à un message dans lequel le scripteur demande pourquoi il ne parvient pas à charger de la musique sur son téléphone portable]

Déjà est-ce que ton téléphone est équipé d'une carte micro sd ? (Forum internet, 2009)

Selon le contexte, une paraphrase possible de (4) pourrait être: 'la première chose que je voudrais savoir, c'est si ton téléphone est équipé d'une carte micro sd', ou 'la première question que tu dois te poser, c'est celle de savoir si ton téléphone est équipé d'une carte micro sd'. Ici, déjà annonce que la personne qui essaye de résoudre le problème va vraisemblablement poser une (au moins) autre question, et que les réponses qui seront apportées à ces questions devraient permettre de trouver la solution au problème technique posé par l'usager. C'est dans cette orientation fonctionnelle commune des questions que réside le lien avec l'argumentation.

Au plan syntaxique, ce statut méta-énonciatif en fait un élément extra-propositionnel. On peut donc dire, dans les termes de la macro-syntaxe élaborée par Alain Berrendonner (à par. Chap. 8), que déjà justificatif constitue à lui seul une clause syntaxique, si bien que les énoncés comportant cet emploi de déjà forment des périodes bi-clausales, sur le même modèle que Sincèrement, il lui a parlé (interprété comme 'Je te le dis sincèrement : il lui a parlé', et non comme 'Il lui a parlé avec sincérité'). Cette extériorité de déjà le dégage des contraintes qui pèsent sur lui lorsqu'il est sous la dépendance du prédicat verbal. Par exemple, c'est un fait bien connu que quand déjà modifie ce prédicat (par exemple en interprétation " de survenance précoce »), il s'accommode très mal de la négation (ne)...pas (cf. ? Il n'est pas déjà sorti); mais cette contrainte disparaît quand il est externe, comme le montrent les exemples (2) et (3) discutés plus haut. Il a également été observé que déjà " de survenance précoce » accepte difficilement les énoncés gnomiques (König 1977, pour l'all. schon, mais ses observations valent aussi pour déjà), ce qui est d'ailleurs assez compréhensible, compte tenu du caractère en principe atemporel de ce type d'énoncé. Or cette contrainte disparaît, elle aussi, quand il est en emploi justificatif. Par exemple, à quelqu'un qui prétendrait que l'éléphant d'Asie et l'éléphant d'Afrique sont difficiles à distinguer, on pourrait rétorquer quelque chose comme: Au contraire c'est très facile. Déjà, l'éléphant d'Asie a des oreilles beaucoup plus petites que l'éléphant d'Afrique. 
4. Au total, on peut donc considérer que la séquence argumentative que produit déjà justificatif comporte, dans sa forme discursive complète, les quatre éléments suivants :

- une première justification (J1), sur laquelle porte déjà ;

- une seconde justification (J2) de même orientation argumentative que J1 et vis-à-vis de laquelle $\mathrm{J} 1$ se voit attribuer une certaine priorité ;

- un corrélateur, introduisant $\mathrm{J} 2$ et liant $\mathrm{J} 2$ à $\mathrm{J} 1$ relativement à la conclusion ;

- une conclusion (C), que J1 et J2 sont censés étayer ou accréditer.

L'exemple suivant présente une séquence complète :

(5) - La ministre de la Santé assure que nous sommes prêts à faire face à une éventuelle pandémie.

D.R. : Tout en ayant un vaccin à disposition, on ne sait déjà pas gérer la grippe saisonnière en France! Il y a chaque année plus de 2 millions de cas de grippe et 5000 morts dans notre pays, alors imaginez ce qui peut se passer avec un nouveau variant et sans vaccin. On court à la catastrophe. (Site La Provence.com)

La réponse que fait D.R., spécialiste en virologie, au journaliste, consiste tout d'abord à formuler J1 ('on ne sait pas gérer la grippe saisonnière en France'), puis - mais pas immédiatement - J2, introduit par le corrélateur alors ('imaginez ce qui peut se passer avec un nouveau variant et sans vaccin'). La conclusion $\mathrm{C}$ est ici donnée en fin de séquence ('on court à la catastrophe'), et constitue en quelque sorte l'élément central de la réponse. Elle pourrait d'ailleurs tout aussi bien figurer en tête de la réponse, comme dans la formulation suivante :

(5') On court à la catastrophe: on ne sait déjà pas gérer la grippe saisonnière en France, alors imaginez ce qui peut se passer avec un nouveau variant et sans vaccin.

Comme on le voit, déjà justificatif induit, en raison de ses implications argumentatives, toute une séquence discursive, admettant plusieurs variantes de formulations et dont certains éléments peuvent être omis (la seconde justification, la conclusion, le corrélateur). Le cas échéant, la formulation est susceptible de solliciter toutes sortes de contenus implicites.

\section{Variantes positionnelles}

Examinons rapidement les principales variantes positionnelles de cet emploi de déjà.

- Son statut méta-énonciatif est souvent souligné par une position initiale et détachée, comme dans (1), (2) et (4), ou par une position finale en "appendice », comme dans l'exemple suivant :

(6) Je vous demanderai d'abord, monsieur, pourquoi vous me mêlez si obstinément à la collection Campana, en affirmant qu'elle a été ma ressource, mon inspiration permanente? Or j'avais fini Salammbô au mois de mars, six semaines avant l'ouverture de ce musée. Voilà une erreur, déjà. (Flaubert, Corresp., 1865, F) 
- En position initiale, il peut prendre la forme d'une expression rectrice de complétive (déjà que...), comme dans (2). Il serait évidemment intéressant d'examiner s'il y a des différences sémantiques entre «déjà $\mathrm{P}$ » et «déjà que $\mathrm{P}$ »- chose impossible à faire dans le cadre de cet article. Mosegaard Hansen (2008, Chap. 7) relève plusieurs différences, touchant à la force justificative et au statut informatif (présupposition) du contenu sur lequel porte déjà, mais ses conclusions sur ces deux points ne nous paraissent pas complètement convaincantes. Cet auteur mentionne cependant un troisième point qui mérite d'être noté : déjà que, contrairement à déjà tout court, est presque toujours orienté vers des conclusions "détrimentales », c'est-à-dire dont le contenu est considéré comme non souhaitable par l'énonciateur (ou par une autre instance, par exemple l'énonciataire) ${ }^{6}$. Petit échantillon d'exemples trouvés sur Frantext, dont nous ne donnons ici que l'amorce, en elle-même suffisamment évocatrice :

(7) déjà que je n'avance pas...

déjà que ça coûte ultra cher de surfer en $3 \mathrm{G} . .$.

déjà que notre cote n'était pas brillante...

déjà que ce n'est jamais folâtre $[. .$.$] de veiller les morts...$

déjà que s'endormir n'est pas drôle...

déjà que ce salaud ne me laisse pas souvent les voir...

déjà que c'est plein d'obèses aux States...

déjà que j'étais cataloguée snobarde...

déjà qu'on est tous des artistes ratés...

déjà que l'Europe traverse une crise sans précédent...

- Mais déjà justificatif peut également être inséré dans la proposition, comme dans (3), (5) et dans les exemples ci-dessous, ce qui ne l'empêche pas - notons-le - de conserver son statut méta-énonciatif.

(8) On ne sait déjà pas quand on commencera à travailler, alors savoir quand on partira à la retraite... (Doc. internet)

(9) Ce que je voudrais savoir, c'est la façon de porter plainte sur Twitter contre abus à autrui $[\ldots]$... Est-ce que déjà c'est possible ? (Forum internet)

Cette insertion semble particulièrement fréquente dans les $\mathrm{P}$ subordonnées ou coordonnées, probablement par un effet de concurrence positionnelle avec la conjonction :

(10) - Pourquoi la vie est-elle apparue?

- Je préfère "comment" à "pourquoi", car on ne sait déjà pas comment la vie est apparue, comment s'est effectué le passage de l'inanimé à l'animé. Parler du "pourquoi", c'est postuler une sorte de finalité de la vie, ce qui reste à prouver. (Doc. internet)

(11) - A qui la faute, sinon à lui ? Alors que j'étais déjà pauvre, je me suis saigné aux artères pour qu'il fasse des études. (Aymé, 1950, F)

\footnotetext{
${ }^{6} \mathrm{D}$ 'où le fait que déjà que introduit très souvent une $\mathrm{P}$ négative. Cela dit, nous avons quelques exemples dans lesquels déjà que n'a rien de détrimental, ce qui montre que ce problème mériterait un examen plus approfondi.
} 
- Déjà justificatif peut également figurer dans des compléments prépositionnels, notamment à valeur justificative, comme : à cause de..., du fait que... En ce cas, la conclusion est donnée dans la proposition dont dépend le complément :

(12) En conclusion, la Smart est toujours aussi smart, déjà à cause de son prix - à partir de 17700 euros - et de son côté décalé, attachant même... (Doc. internet)

(13) Nous voilà donc en présence d'un jeu très intéressant, déjà du fait qu'on puisse incarner le « King of The Pop ». (Journal internet, 2010)

\section{Structures discursives}

Les éléments identifiés ci-dessus $(\mathrm{C}, \mathrm{J} 1, \mathrm{~J} 2$, corrélateur) permettent de décrire les différentes structures discursives que peut induire la présence d'un déjà justificatif. Le principal point à prendre en considération est la position respective de $\mathrm{J} 1$ et $\mathrm{J} 2$, qui conditionne l'apparition du corrélateur. Les cas suivants ont été observés :

- absence de $\mathrm{J} 2$

- ordre $\mathrm{J} 1-\mathrm{J} 2$

- ordre $\mathrm{J} 2-\mathrm{J} 1$

- ordre J2-J1-J2' (J2' étant une version reformulée de J2).

Examinons rapidement ces différents cas.

Absence de J2. - Elle est fréquente, mais difficile à «prouver » exemple à l'appui : il est en effet toujours possible de soupçonner qu'elle n'est qu'un artefact de la manière dont l'extrait a été découpé, et que J2 vient peut-être «plus loin » ou «avant». Disons que (3), repris ci-dessous, pourrait en être une illustration.

(3) C. Allègre ne maitrisant déjà pas l'acronyme G.I.E.C., faut-il porter attention au reste de son ouvrage? (Titre de rubrique, journal Internet)

L'absence de J2 est l'une des causes de l'effet de « suspens » qui accompagne beaucoup de déjà justificatifs (dans les documents écrits, les points de suspension sont nombreux aux alentours de cet emploi de déjà).

Ordre J1-J2. - On l'observe dans plusieurs des exemples déjà commentés : (1), avec le corrélateur encore; (5) et (8), avec le corrélateur alors; (10), sans corrélateur. L'exemple (12) est ambigu, car on ne sait pas si la séquence et de son côté décalé, attachant même est incluse dans la portée de déjà (autrement dit, dans J1), ou si elle désigne J2. En voici un autre exemple, avec encore comme corrélateur :

(14) «La nostalgie vient de l'incapacité à haïr» me dit F. M'a-t-elle destiné la formule ? Si j'ai déjà bien du mal à me sentir objet de haine, j'ai encore beaucoup plus de mal à me reconnaître sujet haïssant; à vrai dire je n'y parviens pas. (Pontalis, 2002, F)

On interprétera cet exemple de la façon suivante : j'ai bien du mal à me sentir objet de haine est marqué par déjà comme la justification $\mathrm{J} 1$; la séquence j'ai beaucoup plus de mal à 
me reconnaître sujet haïssant; à vrai dire je n'y parviens pas est marquée par encore comme la justification $\mathrm{J} 2$. La conclusion vers laquelle sont orientées ces deux justifications pourrait être construite comme : 'la haine est un sentiment qui m'est étranger (aussi bien comme objet haï que comme sujet haïssant)'.

- J1 : 'j'ai bien du mal à me sentir objet de haine'

- J2 : 'j'ai beaucoup plus de mal à me reconnaître sujet haïssant; à vrai dire je n'y parviens pas'

- C : non formulé. Par exemple: 'la haine est un sentiment auquel je suis étranger (aussi bien comme objet haï que comme sujet haïssant)'

- Corrélateur : encore

Ordre J2-J1. - On l'observe dans l'extrait suivant :

(15) L'Abbé : [...] Ce qu'il faut, c'est que vous disparaissiez totalement.

Sevrais : Comment cela?

L'Abbé : Il ne faut plus revoir du tout Soubrier.

Sevrais : Quoi ! Quand déjà, si je restais deux jours sans le voir... mais non, ce n'est pas cela que vous voulez dire !...

L'Abbé : C'est cela.

(Montherlant, 1951, F)

Cet extrait est assez délicat à analyser, car tous les éléments doivent plus ou moins être reconstruits. J1 y est ouvertement allusif, incomplet : Quand déjà, si je restais deux jours sans le voir... On pourrait l'extrapoler par une formulation comme: 'je supportais très mal de rester deux jours sans le voir'. $\mathrm{J} 2$ doit être construit à partir de la réplique $I l$ ne faut plus revoir du tout Soubrier, et pourrait être formulé ainsi : 'vous me demandez de ne plus revoir Soubrier'. Quant au contenu de C, il est complètement implicite et pourrait correspondre par exemple à une formulation comme : 'vous me demandez quelque chose d'impossible'. Soit, si on reconstruit le tout comme un monologue :

(15') Vous me demandez de ne plus revoir du tout Soubrier. C'est impossible. Déjà, je supportais très mal de rester deux jours sans le voir.

Par comparaison avec le cas précédent, on pourrait dire ici que déjà - plus exactement le segment comportant déjà -, parce qu'il renvoie à une autre justification antérieurement formulée, du moins à un fait auquel ce statut de justification est attribué, a une dimension anaphorique.

Voici un exemple non dialogal, lui aussi anaphorique au sens ci-dessus :

(16) Ce jour-là, donc, pas un mot à poser sur le papier. On serait derniers, c'est sûr. C'était le redoublement assuré. Déjà que notre cote n'était pas brillante... Je fous un coup de coude et dix coups de tatane dans les chevilles de mon voisin [...] (Bayon, 1987)

On a ici :

- J2 : 'ce jour-là, pas un mot à poser sur le papier'

- C : 'on serait derniers, c'est sûr', ou : 'c 'était le redoublement assuré'

- J1 : 'notre cote n'était pas brillante' 
L'idée de gradient signalée plus haut, dont découle la notion de "priorité » impliquée par déjà, coïncide ici avec l'ordre temporel : notre cote n'était pas brillante désigne en quelque sorte l'arrière-plan situationnel dans lequel s'inscrit l'événement consistant à ne pas poser un seul mot sur le papier.

Ordre J2-J1-J2'. - C'est le cas où, comme précédemment, J2 est formulé avant la proposition comportant déjà $(\mathrm{J} 1)$, et reformulé après cette dernière (d'où $\mathrm{J} 2$ '). Telle est l'analyse qu'on peut donner de l'extrait ci-dessous :

(17) [Propos tenus par un publicitaire :] Au bout d'un certain nombre de créations refusées, on devient complètement désabusé, même si on fait semblant de s'en foutre, ça nous ronge. Déjà qu'on est tous des artistes ratés, en plus on nous force à ravaler notre amour-propre et remplir nos tiroirs avec des maquettes jetées. (ex. repris de Métrich et al., à par.)

Il nous semble en effet qu'on peut interpréter la séquence (en plus) on nous force à [...] remplir nos tiroirs avec des maquettes jetées comme une reformulation de la séquence $A u$ bout d'un certain nombre de créations refusées. Autrement dit, évoquer le fait que "beaucoup de nos créations sont refusées" ou le fait que "nos tiroirs se remplissent de maquettes jetées ", c'est évoquer la même réalité, mais sous des manifestations différentes. Cette réalité justifie le constat suivant lequel on devient complètement désabusé, ..., ça nous ronge (C). Déjà introduit une justification supplémentaire à ce désenchantement : on est tous (considérés comme) des artistes ratés (J1). D’où l'analyse :

- C : 'dans ce métier on devient complètement désabusé, ..., ça nous ronge'

- J2 : 'on essuie souvent des refus'

- $\mathrm{J} 1$ : 'on est tous des artistes ratés'

- J2' : 'on nous force à ravaler notre amour-propre et remplir nos tiroirs avec des maquettes jetées'

- Corrélateur : en plus

Si cette analyse est correcte, on peut considérer que $\mathrm{J} 2$ ' fonctionne ici comme une métalepse ou une métonymie temporelle.

\section{En guise de conclusion}

Parce qu'il est fondamentalement argumentatif, l'emploi de déjà examiné dans cet article induit une séquence discursive relativement complexe. Cette séquence est «à géométrie variable », car elle admet une multitude de variantes de formulations : d'une part, l'ordre des éléments qui la constituent n'est pas fixe ; d'autre part, certains de ces éléments peuvent être omis (la justification secondaire, le corrélateur, la conclusion). Au plan du discours, déjà justificatif participe ainsi d'un petit " programme d'actions communicatives »pour reprendre une expression utilisée par Alain Berrendonner dans sa Grammaire de la période. Il est donc associé à des unités discursives de type "périodes », mais en un sens différent de celui évoqué plus haut, et vraisemblablement plus proche de la période au sens où l'entendait la rhétorique classique. 


\section{Références}

Anscombre J.-C., Ducrot O. (1976). L'argumentation dans la langue. Langages, 42, 5-27.

Apothéloz D., Nowakowska M. (à par.). «Déjà » et le sens des énoncés. Cahiers Chronos. Amsterdam : Rodopi.

Berrendonner A., ss la dir. de (à par.). Grammaire de la période.

Buchi E. (2007). Approche diachronique de la (poly)pragmaticalisation de français déjà. In : Trotter D. (éd.), Actes du XXIV Congrès international de Linguistique et de Philologie Romanes (Aberystwyth), Tübingen: Niemeyer, 251-264.

Comrie B. (1976). Aspect. Cambridge : Cambridge University Press.

Ducrot O. (1980). Les échelles argumentatives. Paris : Minuit.

Fernandez M.M.J. (1994). Les particules énonciatives dans la construction du discours. Paris : Presses Universitaires de France.

Franckel J.-J. (1989). Etude de quelques marqueurs aspectuels du français. Genève : Droz.

Fuchs C. (1988) Encore, déjà, toujours : de l'aspect à la modalité. In : Tersis N. et Kihm A., (éds), Temps et aspects, Actes du colloque CNRS, Paris, 24-25 octobre 1985, Louvain : Peeters, Paris : SELAF, 136-148.

Hoepelman J., Rohrer C. (1980). Déjà, encore et les temps du passé en français. In : David J. et Martin R (éds), La notion d'aspect. Metz: Centre d'Analyse syntaxique de l'Université de Metz (coll. Recherches linguistiques V), p. 119-143.

König E. (1977). Temporal and non-temporal uses of 'noch' and 'schon' in German. Linguistics and Philosophy, 1, 173-198.

Martin R. (1980). Déjà et encore : de la présupposition à l'aspect. In : David J. et Martin R (éds), La notion d'aspect, (coll. Recherches linguistiques V), Metz: Centre d'Analyse syntaxique de l'Université de Metz, p. 167-179.

Métrich R., Faucher E. (2009). Wörterbuch deutscher Partikeln. Unter Berücksichtigung ihrer französischen Äquivalente. (In Zusammenarbeit mit J. Albrecht). Berlin, New York : Walter de Gruyter.

Métrich R., Faucher E., Albrecht J. (à par.). Projet de dictionnaire français-allemand des "mots du discours".

Mosegaard Hansen M.-B. (2002). From Aspectuality to Discourse Marking: the Case of French déjà and encore. Belgian Journal of Linguistics, 16, 23-51.

Mosegaard Hansen M.-B. (2008). Particles at the semantics/pragmatics interface: synchronic and diachronic issues. A study with special reference to the French phasal adverbs. Howard House, UK: Emerald.

Muller C. (1975). Remarques syntactico-sémantiques sur certains adverbes de temps. Le Français moderne, 43, 12-38.

Nowakowska M., Apothéloz D. (2011). Note sur l'adverbe temporel już et ses correspondants français. Cognitive Studies / Etudes Cognitives, 11, 13-30.

Nøjgaard M. (1992-95). Les adverbes français. Essai de description fonctionnelle, 3 vol. Copenhagen : Munksgaard.

Paillard D. (1992). Déjà et la construction de l'énoncé. L'information grammaticale, 55, 3337.

Paillard D. (s.d.). Déjà : adverbe ou marqueur discursif? Communication présentée au colloque Chronos 6, Genève, 2004. Document téléchargeable à l'adresse $<$ www.unige.ch/lettres/latl/chronos/paillard.rtf $>$.

Tahara I. (2006). Adverbes temporels et point de vue : le cas de déjà et bientôt. TRANEL (Travaux neuchâtelois de linguistique), 45, 99-113.

Van der Auwera J. (1993). 'Already' and 'still': beyond duality. Linguistics and Philosophy, 16, 613-653. 